\title{
AFFINE PROJECTIVE OSSERMAN STRUCTURES
}

\author{
P. GILKEY AND S. NIKČEVIĆ
}

\begin{abstract}
By considering the projectivized spectrum of the Jacobi operator, we introduce the concept of projective Osserman manifold in both the affine and in the pseudo-Riemannian settings. If $\mathrm{M}$ is an affine projective Osserman manifold, then the modified Riemannian extension metric on the cotangent bundle is both spacelike and timelike projective Osserman. Since any rank 1 symmetric space is affine projective Osserman, this provides additional information concerning the cotangent bundle of a rank 1 Riemannian symmetric space with the modified Riemannian extension metric. We construct other examples of affine projective Osserman manifolds where the Ricci tensor is not symmetric and thus the connection in question is not the Levi-Civita connection of any metric. If the dimension is odd, we use methods of algebraic topology to show the Jacobi operator of an affine projective Osserman manifold has only one non-zero eigenvalue and that eigenvalue is real.
\end{abstract}

\section{INTRODUCTION}

1.1. Osserman geometry in the Riemannian setting. Let $\mathcal{R}$ be the curvature operator of a Riemannian manifold $\mathcal{M}:=(M, g)$ of dimension $m$. The Jacobi operator $\mathcal{J}(x): y \rightarrow \mathcal{R}(y, x) x$ is a self-adjoint endomorphism of the tangent bundle. Following the seminal work of Osserman 23, one says that $\mathcal{M}$ is Osserman if the eigenvalues of $\mathcal{J}$ are constant on the unit sphere bundle

$$
S(M, g):=\{\xi \in T M: g(\xi, \xi)=1\} .
$$

Work of Chi 10], of Gilkey, Swan, and Vanhecke [18, and of Nikolayevsky [19, 20] shows that any complete and simply connected Osserman manifold of dimension $m \neq 16$ is a rank 1-symmetric space; the 16 dimensional setting is exceptional and the situation is still not clear in that setting although there are some partial results due, again, to Nikolayevsky [21].

There has been much activity recently in Osserman Geometry. Brozos-Vázquez and E. Merino 1 showed that in dimension 4, the Osserman condition and the Rakić duality principle are equivalent. Nikolayevsky 22 showed that a conformally Osserman manifold (here one uses the Weyl conformal tensor to define the Jacobi operator) is locally isometric to a rank-one symmetric space in dimension 16 modulo a certain assumption on algebraic curvature tensors in dimension 16 . Brozos-Vázquez et. al [3] have examined conformally Osserman manifolds using warped product structures.

1.2. Osserman geometry in the pseudo-Riemannian geometry. Suppose that $\mathcal{M}=(M, g)$ is a pseudo-Riemannian manifold of signature $(p, q)$ for $p>0$ and $q>0$. The pseudo-sphere bundles are defined by setting:

$$
S^{ \pm}(M, g)=\{\xi \in T M: g(\xi, \xi)= \pm 1\} .
$$

One says that $(M, g)$ is spacelike (resp. timelike) Osserman if the eigenvalues of $\mathcal{J}$ are constant on $S^{ \pm}(M, g)$. The situation is rather different here as the Jacobi

2000 Mathematics Subject Classification. 53C50, 53C44.

Key words and phrases. affine Osserman, affine projective Osserman, spacelike projective Osserman, timelike projective Osserman. 
operator is no longer diagonalizable and can have nontrivial Jordan normal form as shown by García-Río et al [13]; in the algebraic context, the Jordan normal form can be arbitrarily complicated [17]. One says $(M, g)$ is nilpotent if $\mathcal{J}(x)$ is nilpotent for any tangent vector $x$; this does not imply $(M, g)$ is flat in the pseudo-Riemannian setting.

Even in signature $(2,2)$, the situation is far from clear although much progress has been made recently by Calviño-Louzao et al [6] in examining these questions and similar questions related to the skew-symmetric curvature operator and by Díaz-Ramos et al 14 in examining non-diagonalize Jacobi operators. Derdzinski 11] has examined questions concerning type III Jordan-Osserman metrics raised by Diaz-Ramos et al [12. Walker geometry is intimately related with many questions in mathematical physics. Chaichi et. al. 7] have studied conditions for a Walker metric to be Einstein, Osserman, or locally conformally flat and obtained thereby exact solutions to the Einstein equations for a restricted Walker manifold. Chudecki and Prazanowski [8, 9] examined Osserman metrics in terms of 2-spinors and provided some new results in HH-geometry using the close relation between weak HH-spaces and Walker and Osserman spaces using results of [12.

1.3. Affine Osserman manifolds. Let $\nabla$ be a torsion free connection on a smooth manifold $M$; the pair $(M, \nabla)$ is said to be an affine manifold. The first work on Osserman geometry in the affine setting is due to García-Río et al 16. One has $\mathcal{J}(\lambda x)=\lambda^{2} \mathcal{J}(x)$ for $\lambda \in \mathbb{R}$. This rescaling must be taken into effect. If $T$ is a linear map of a finite dimensional real vector space, let $\operatorname{Spec}\{T\} \subset \mathbb{C}$ be the spectrum of $T$; this is the set of roots of the characteristic polynomial $P_{T}(\lambda):=\operatorname{det}(T-\lambda \mathrm{Id})$. One says that an affine manifold $(M, \nabla)$ is affine Osserman if $\operatorname{Spec}(\mathcal{J}(x))=\{0\}$ for any tangent vector $x$; i.e. $\mathcal{J}(x)$ is nilpotent. This notion clearly is invariant under rescaling and there are many examples. One has, for example, the following result of García-Río et al [13:

Theorem 1.1. Define a torsion free connection on $\mathbb{R}^{m}$ by setting

$$
\nabla_{\partial_{x_{i}}} \partial_{x_{j}}=\sum_{k>\max (i, j)} \Gamma_{i j}{ }^{k}\left(x_{1}, \ldots, x_{k-1}\right) \partial_{x_{k}} \text { for } \Gamma_{i j}{ }^{k}=\Gamma_{j i}{ }^{k} \text {. }
$$

Then $(M, \nabla)$ is affine Osserman.

Such examples are important in neutral signature Osserman geometry. Let $(M, \nabla)$ be an affine manifold. Let $\left(x^{1}, \ldots, x^{m}\right)$ be local coordinates on $M$. If $\omega \in T^{*} M$, expand $\omega=\sum_{i} y_{i} d x^{i}$ to define the dual fiber coordinates $\left(y_{1}, \ldots, y_{m}\right)$ and thereby obtain canonical local coordinates $\left(x^{1}, \ldots, x^{m}, y_{1}, \ldots, y_{m}\right)$ on $T^{*} M$. Let $\Phi=\Phi_{i j} d x^{i} \circ d x^{j}$ be a smooth symmetric 2 -tensor on $M$. The deformed Riemannian extension $g_{\nabla, \Phi}$ is the metric of neutral signature $(\bar{m}, \bar{m})$ on the cotangent bundle $T^{*} M$ given locally by

$$
\begin{aligned}
& g_{\nabla, \phi}\left(\partial_{x_{i}}, \partial_{x_{j}}\right)=-2 y_{k} \Gamma_{i j}{ }^{k}(x)+\Phi_{i j}(x), \\
& g_{\nabla, \phi}\left(\partial_{x_{i}}, \partial_{y^{j}}\right)=\delta_{i}^{j}, \quad g_{\nabla, \phi}\left(\partial_{y^{i}}, \partial_{y^{j}}\right)=0 .
\end{aligned}
$$

This is invariantly defined; we refer to Calvino-Louzao et al [4] for further details. One has:

Theorem 1.2. Let $(M, \nabla)$ be an affine Osserman manifold and let $\Phi$ be a smooth symmetric 2 -tensor on $M$. Then the deformed Riemannian extension $\left(T^{*} M, g_{\nabla, \Phi}\right)$ is a pseudo-Riemannian nilpotent Osserman manifold of neutral signature.

It is possible to modify this construction to produce Osserman metrics with nonnilpotent Jacobi operators of neutral signature on $T^{*} M$ Calvino-Louzao et al [5]. 
One defines the modified Riemannian extension by setting:

$$
\begin{aligned}
& g_{\nabla, 1}\left(\partial_{x_{i}}, \partial_{x_{j}}\right)=-2 y_{k} \Gamma_{i j}{ }^{k}(x)+y_{i} y_{j}, \\
& g_{\nabla, 1}\left(\partial_{x_{i}}, \partial_{y^{j}}\right)=\delta_{i}^{j}, \quad g_{\nabla, 1}\left(\partial_{y^{i}}, \partial_{y^{j}}\right)=0 .
\end{aligned}
$$

Again, this is invariantly defined. One has:

Theorem 1.3. Let $(M, \nabla)$ be an affine Osserman manifold. Then the modified Riemannian extension $\left(T^{*} M, g_{\nabla, 1}\right)$ is a pseudo-Riemannian Osserman manifold of neutral signature so that if $\xi_{ \pm} \in S^{ \pm}\left(T^{*} M, g_{\nabla, 1}\right)$, then $\operatorname{Spec}\left\{\mathcal{J}\left(\xi_{ \pm}\right)\right\}= \pm\left(0,1, \frac{1}{4}\right)$ with multiplicities $(1,1,2 m-2)$, respectively.

Note that the structures can be chosen so that Jacobi operators for the metrics in Theorem 1.2 and and in Theorem 1.3 have non-trivial Jordan normal form.

1.4. Projectivizing the spectrum. Since $\mathcal{J}(\lambda \xi)=\lambda^{2} \mathcal{J}(\xi)$, it is necessary to take this rescaling into account. This played no role, of course, if we assume that $\operatorname{Spec}\{\mathcal{J}(\xi)\}=\{0\}$ for all $\xi$. But it motivates the following:

\section{Definition 1.4.}

(1) Let $(M, \nabla)$ be an affine manifold. We say $(M, \nabla)$ is an affine projective Osserman manifold if given any pair of non-zero tangent vectors $x, y$, there is a real scaling factor $s(x, y) \neq 0$ so

$$
\operatorname{Spec}\{\mathcal{J}(y)\}=s(x, y) \cdot \operatorname{Spec}\{\mathcal{J}(x)\} \neq\{0\} .
$$

(2) Let $(M, g)$ be a pseudo-Riemannian manifold. We say $(M, g)$ is spacelike projective Osserman (resp. timelike projective Osserman) if given any pair of vectors $x, y$ in $S^{+}(M, g)$ (resp. $S^{-}(M, g)$ ), there is a real scaling factor $s(x, y) \neq 0$ so

$$
\operatorname{Spec}\{\mathcal{J}(y)\}=s(x, y) \cdot \operatorname{Spec}\{\mathcal{J}(x)\} \neq\{0\} .
$$

Although in principle, we allowed $s(x, y)$ to be negative, in fact $s(x, y)$ can be chosen to be positive and once this is done, $s$ is smooth. We will establish the following result in Section 2,

Lemma 1.5. Let $(M, \nabla)$ be an affine manifold. Let $\mathcal{O}$ be a connected open subset of TM. Suppose there exists $s(x, y)$ so that $\operatorname{Spec}\{\mathcal{J} x)\}=s(x, y) \operatorname{Spec}\{\mathcal{J}(y)\} \neq\{0\}$ for all $x, y \in \mathcal{O}$. Then:

(1) $\operatorname{Tr}\left\{\mathcal{J}(x)^{k}\right\}=s(x, y)^{k} \operatorname{Tr}\left\{\mathcal{J}(y)^{k}\right\}$ for any $x, y \in \mathcal{O}$ and any $k$.

(2) $\operatorname{Spec}\{\mathcal{J}(x)\}=|s(x, y)| \operatorname{Spec}\{\mathcal{J}(x)\} \neq\{0\}$ for all $x, y \in \mathcal{O}$.

(3) There exists $k$ so that

$$
|s(x, y)|=\left\{\frac{\operatorname{Tr}\left\{\mathcal{J}(x)^{k}\right\}}{\operatorname{Tr}\left\{\mathcal{J}(y)^{k}\right\}}\right\}^{1 / k} \quad \text { for any } x, y \in \mathcal{O} .
$$

(4) The function $|s(x, y)|$ is smooth on $\mathcal{O} \times \mathcal{O}$.

Since $S^{ \pm}(M, g)$ has at most two components and since $\mathcal{J}(-\xi)=\mathcal{J}(\xi)$, the following result is an immediate consequence of Lemma 1.5

\section{Theorem 1.6.}

(1) Let $(M, \nabla)$ be an affine projective Osserman manifold. Then the function $s(x, y)$ of Definition 1.4 (1) can be taken to be positive and smooth.

(2) Let $(M, g)$ be a spacelike projective Osserman (resp. timelike projective Osserman) manifold. Then the function $s(x, y)$ in Definition 1.4 (2) can be taken to be positive and smooth. 
The notions of timelike Osserman and spacelike Osserman are equivalent (see García-Río et al [15]). This is not the situation in the setting at hand as we shall show in Section 3 .

Theorem 1.7. Let $p>0$ and $q>0$. There exists a pseudo-Riemannian manifold $(M, g)$ of signature $(p, q)$ which is spacelike projective Osserman but not timelike projective Osserman. Similarly, there exists a pseudo-Riemannian manifold $(\tilde{M}, \tilde{g})$ of signature $(p, q)$ which is timelike projective Osserman but not spacelike projective Osserman.

In Section 4, we will generalize Theorem 1.2 to the projective setting:

Theorem 1.8. Let $\Phi$ be a symmetric 2 tensor on an affine manifold $(M, \nabla)$. The following assertions are equivalent:

(1) $(M, \nabla)$ is an affine projective Osserman manifold.

(2) $\left(T^{*} M, g_{\nabla, \Phi}\right)$ is a spacelike projective Osserman manifold.

(3) $\left(T^{*} M, g_{\nabla, \Phi}\right)$ is timelike projective Osserman manifold.

Let $\rho(x, y):=\operatorname{Tr}\{z \rightarrow R(z, x) y\}$ be the Ricci tensor. This tensor need no longer be symmetric so we let $\rho_{s}(x, y):=\frac{1}{2}\{\rho(x, y)+\rho(y, x)\}$ be the symmetric part of this tensor. Any Riemannian Osserman manifold is necessarily an affine projective Osserman manifold; the fact that $(M, g)$ is Riemannian is crucial here since if $\mathcal{J}(\xi)$ is nilpotent if $\xi$ is null for a higher signature pseudo-RiemannianOsserman manifold. Consequently if $(M, g)$ is a rank 1 symmetric space, then $(M, g)$ is an affine projective Osserman manifold. If $m=2$ and if $0 \neq x$, let $\{0, \lambda(x)\}$ be the eigenvalues of $\mathcal{J}(x)$ where each eigenvalue is repeated according to its multiplicity. Then $\rho(x, x)=\rho_{s}(x, x)=\operatorname{Tr}\{\mathcal{J}(x)\}=\lambda(x)$. The following result is now immediate and provides examples to which Theorem 1.8 applies:

\section{Theorem 1.9.}

(1) Any rank 1-symmetric space is an affine projective Osserman manifold where we let $\nabla$ be the Levi-Civita connection.

(2) If $m=2$ and if $(M, \nabla)$ is an affine manifold, then $(M, \nabla)$ is an affine projective Osserman manifold if and only if $\rho_{s}(x, x) \neq 0$ for all $x$, i.e. $\rho_{s}$ is definite.

1.5. The algebraic context. Let $V$ be a real vector space of dimension $m$ and let $A \in \operatorname{End}(V) \otimes V^{*}$. We say that $(V, A)$ is an affine curvature model if $A$ has the symmetries of the curvature operator of an affine connection for all $x, y, z \in V$ :

$$
\begin{aligned}
& A(x, y) z=-A(y, x) z \\
& A(x, y) z+A(y, z) x+A(z, x) y=0 .
\end{aligned}
$$

The first symmetry is the $\mathbb{Z}_{2}$ anti-symmetry and the second symmetry is the first Bianchi identity. If $(M, \nabla)$ is an affine manifold, then $\left(T_{P} M, R_{P}\right)$ is an affine curvature model for any $P \in M$. Conversely, given an affine curvature model $(V, A)$, then there exists a complete affine manifold $(M, \nabla)$ and a point $P$ of $M$ so that $(V, A)$ is isomorphic to $\left(T_{P} M, R_{P}\right)$, i.e. every affine curvature model can be geometrically realized by a complete affine manifold (see Y. Euh et al [2]).

Let $(V, A)$ be an affine curvature model. The associated Jacobi operator is given by setting $\mathcal{J}(v) w:=A(w, v) v$. One says that $(V, A)$ is an affine projective Osserman curvature model if $\operatorname{Spec}\{\mathcal{J}(v)\}=s(v, w) \operatorname{Spec}\{\mathcal{J}(w)\} \neq\{0\}$ for $0 \neq v, w \in V$. In Section 5] we will prove the following result which has an immediate application to the geometric setting:

Theorem 1.10. Let $(V, A)$ be a an affine projective Osserman curvature model of odd dimension $m$. If $0 \neq v \in V$, then $\operatorname{Spec}\{\mathcal{J}(v)\}=\{0, \lambda(v)\}$ where $\lambda(v)$ is a 
smooth real valued function on $V-\{0\}$ which never vanishes. The eigenvalue 0 appears with multiplicity 1 and the eigenvalue $\lambda(v)$ appears with multiplicity $m-1$. In this situation $\rho(v, v)=(m-1) \lambda(v)$ so the symmetric Ricci tensor $\rho_{s}$ defines a non-degenerate definite inner product on $V$.

In Section 6, we will prove the following result:

Theorem 1.11. Let $\mathfrak{M}_{\varepsilon}:=\left(\mathbb{R}^{m}, A\right)$ where the non-zero components of $A$ are determined by:

$$
A_{i j j}{ }^{i}=1 \text { for } 1 \leq i \neq j \leq m \text { and } A_{122}{ }^{2}=A_{121}{ }^{1}=-\varepsilon .
$$

(1) $\mathfrak{M}_{\varepsilon}$ is an affine projective Osserman model for any $\epsilon$.

(2) $\mathfrak{M}_{\varepsilon}$ is geometrically realizable by an affine projective Osserman manifold.

Remark 1.12. The Ricci tensor of the model in Theorem 1.11 is given by

$$
\rho\left(e_{i}, e_{j}\right)=\left\{\begin{aligned}
\varepsilon & \text { if } i=1, j=2 \\
-\varepsilon & \text { if } i=2, j=1 \\
m-1 & \text { if } i=j \\
0 & \text { otherwise }
\end{aligned}\right\} .
$$

If $\varepsilon \neq 0$, then $\rho_{s}$ is not symmetric and $A$ is not a Riemannian algebraic curvature operator and, in particular, is not the curvature operator of constant sectional curvature +1 .

The tensor of Theorem 1.11 is a perturbation of the curvature tensor of constant sectional curvature +1 . In Section 7 we present two algebraic examples which are perturbations of the Fubini-Study metric on complex projective space and on quaternionic projective space, respectively, and which are affine projective Osserman models.

\section{The PRoOf of Lemma 1.5}

Let $(M, \nabla)$ be an affine manifold and let $\mathcal{O}$ be an open connected subset of $T M$. Assume $\operatorname{Spec}\{\mathcal{J}(x)\}=s(x, y) \operatorname{Spec}\{\mathcal{J}(y)\} \neq\{0\}$ for all $x, y \in \mathcal{O}$. Let $\sigma(t)$ be a path in $\mathcal{O}$. Since the number of eigenvalues of $\operatorname{Spec}\{\mathcal{J}(\sigma(t))\}$ is independent of $t$, eigenvalues do not coalesce or bifurcate and consequently the eigenvalue multiplicities are constant as well along $\sigma$. Thus

$$
\operatorname{Tr}\left\{\mathcal{J}(t)^{k}\right\}=s(\sigma(0), \sigma(t))^{k} \operatorname{Tr}\left\{\mathcal{J}(0)^{k}\right\} \text { for any } k .
$$

Since $\mathcal{J}(\sigma(0))$ is not nilpotent, $\operatorname{Tr}\left\{\mathcal{J}(\sigma(0))^{k}\right\} \neq 0$ for some $k$. Fix such a $k$. Since $\mathcal{O}$ is connected, Equation (2.a) implies that $\operatorname{Tr}\left\{\mathcal{J}(x)^{k}\right\} \neq 0$ for any $x \in \mathcal{O}$ and that $s(\sigma(0), \sigma(t))^{k}$ is smooth. If $k$ is odd, since $s(\sigma(0), \sigma(0))=1$ and $s(\sigma(0), \sigma(t)) \neq 0$, we have $s(\sigma(0), \sigma(t))>0$. Since the endpoints were arbitrary, $s(x, y)>0$ for all $(x, y)$ and the Lemma follows.

On the other hand, if $\operatorname{Tr}\left\{\mathcal{J}(\sigma(0))^{k}\right\}=0$ for all odd $k$, then $\operatorname{Spec}\{\mathcal{J}(\sigma(0))\}$ is symmetric about the origin and we may assume $s(\sigma(0), \sigma(t))$ is positive. Again, we can take the $k^{\text {th }}$ root to establish Lemma 1.5.

\section{The PRoOf of TheOREM 1.7}

Let $p>0$ and let $q>0$ be given. Let $\left(S^{q}, g_{q}\right)$ denote the sphere in $\mathbb{R}^{q+1}$ with the standard metric of constant sectional curvature +1 . Let $\left(\mathbb{R}^{p}, g_{p}\right)$ denote $\mathbb{R}^{p}$ with a flat negative definite metric. Let $M=\left(\mathbb{R}^{p} \times S^{q}, g_{p} \oplus g_{q}\right)$; this metric has signature $(p, q)$. If $\xi=\left(\xi_{p}, \xi_{q}\right) \in T M$, then $\mathcal{J}(\xi)=0 \oplus \mathcal{J}\left(\xi_{q}\right)$. If $\xi$ is spacelike, then $\xi_{q} \neq 0$ and $\operatorname{Spec}\{\mathcal{J}(\xi)\}=\operatorname{Spec}\left\{\mathcal{J}\left(\xi_{q}\right)\right\}=\left\{0,\left|\xi_{q}\right|^{2}\right\}$ and thus $(M, g)$ is a spacelike projective Osserman manifold; 0 is an eigenvalue of multiplicity $p$. On the other hand, if $\xi_{q}=0$ and $\xi_{p} \neq 0$, then $\xi$ is timelike and $\operatorname{Spec}\{\mathcal{J}(\xi)\}=\{0\}$ so $(M, g)$ 
is not a timelike projective Osserman manifold. This proves the first assertion of Theorem 1.7, the second follows similarly.

\section{The Proof of Theorem 1.8}

Let $\sigma$ be the canonical projection from $T^{*} M$ to $M$. Let $\xi \in T\left(T^{*} M\right)$ and let $a=\sigma_{*} \xi \in T M$. Relative to the canonical frame $\left(\partial_{x_{1}}, \ldots, \partial_{x_{m}}, \partial_{y^{1}}, \ldots, \partial_{y^{m}}\right)$ for $T\left(T^{*} M\right)$, one has (see García-Río et al [16]) that:

$$
\mathcal{J}_{g_{\nabla, \Phi}}(\xi)=\left(\begin{array}{ll}
\mathcal{J}_{\nabla}(a) & 0 \\
* & { }^{t} \mathcal{J}_{\nabla}(a)
\end{array}\right)
$$

where $*$ is some linear map from $\operatorname{Span}\left\{\partial_{x_{i}}\right\}$ to $\operatorname{Span}\left\{\partial_{y^{k}}\right\}$. Consequently

$$
\operatorname{Spec}\left\{\mathcal{J}_{g_{\nabla, \Phi}}(\xi)\right\}=\operatorname{Spec}\left\{\mathcal{J}_{\nabla}(a)\right\} .
$$

If $\xi_{ \pm} \in S^{ \pm}\left(T^{*} M, g_{\nabla, \Phi}\right)$, then $a:=\sigma_{*} \xi_{ \pm} \neq 0$. The implication $(1) \Rightarrow(2)$ and the implication $(1) \Rightarrow(3)$ of Theorem 1.8 now follow. Conversely, suppose that Assertion (2) holds or that Assertion (3) holds. Let $a \neq 0$. Choose $\xi \in S^{ \pm}\left(T^{*} M, g_{\nabla, \Phi}\right)$ so that $\sigma_{*}(\xi)=t a$ for some $t \neq 0$; The implications $(2) \Rightarrow(1)$ and $(3) \Rightarrow(1)$ now follow.

\section{The PRoOF OF TheOREM 1.10]}

Let $(V, A)$ be an affine projective Osserman curvature model. Fix a basepoint $0 \neq x \in V$ and let $\operatorname{Spec}\{\mathcal{J}(x)\}=\left\{0, \lambda_{1}, \ldots\right\}$; by hypothesis $\operatorname{Spec}\{\mathcal{J}(x)\} \neq\{0\}$. If $0 \neq y \in V, \operatorname{Spec}\{\mathcal{J}(y)\}=\left\{0, s(y, x) \lambda_{1}, \ldots\right\}$. Let

$$
V_{1}(y)=\left\{\begin{array}{l}
\operatorname{ker}\left\{\left(\mathcal{J}(y)-s(y, x) \lambda_{1}\right)^{m}\right\} \text { if } \lambda_{1} \in \mathbb{R} \\
\operatorname{ker}\left\{\left(\mathcal{J}(y)-s(y, x) \lambda_{1}\right)^{m}\left(\mathcal{J}(y)-s(y, x) \bar{\lambda}_{1}\right)^{m}\right\} \text { otherwise }
\end{array}\right\}
$$

be the generalized eigenspace corresponding to $\lambda_{1}$ if $\lambda_{1}$ is real and to $\left\{\lambda_{1}, \bar{\lambda}_{1}\right\}$ otherwise. As noted previously, the eigenvalue multiplicities are constant. Thus these generalized eigenspaces have constant dimension and vary smoothly with $y$. Put an auxiliary inner product $\langle\cdot, \cdot\rangle$ on $V$ and let $S^{m-1}$ be the unit sphere of $(V,\langle\cdot, \cdot\rangle)$. Let $y \in S^{m-1}$. Since $\mathcal{J}(y) y=0, \mathcal{J}(y)$ induces an endomorphism of the quotient space $V / y \cdot \mathbb{R}$ which we may identify with $T_{y} S^{m-1}$. Since $m-1$ is even, $S^{m-1}$ has no non-trivial sub-bundles. Since $\{0\} \neq V_{1}$ is a sub-bundle of $T S^{m-1}$, we conclude $V_{1}=T S^{m-1}$ for $y \in S^{m-1}$. This implies that 0 is an eigenvalue of multiplicity 1 and that

$$
\operatorname{Spec}\{\mathcal{J}(y)\}=\left\{\begin{array}{l}
\left\{0, s(y, x) \lambda_{1}\right\} \text { if } \lambda \in \mathbb{R} \\
\left\{0, s(y, x) \lambda_{1}, s(y, x) \bar{\lambda}_{1}\right\} \text { otherwise }
\end{array}\right\} .
$$

This completes the proof if $\lambda_{1}$ is real. Thus we suppose $\lambda_{1}$ is complex and argue for a contradiction. We complexify and decompose

$$
T_{y}\left(S^{m-1}\right) \otimes_{\mathbb{R}} \mathbb{C}=W_{s(y, x) \lambda_{1}} \oplus W_{s(y, x) \bar{\lambda}_{1}}
$$

into the generalized eigenbundles corresponding to $\lambda$ and $\bar{\lambda}$ where

$$
W_{\mu}(y):=\left\{\xi \in T_{y} S^{m-1} \otimes_{\mathbb{R}} \mathbb{C}:(\mathcal{J}(y)-\mu)^{m} \xi=0\right\} .
$$

Since $\mathcal{J}(-y)=\mathcal{J}(y)$, we obtain a corresponding decomposition of the tangent bundle of projective space

$$
T\left(\mathbb{R} P^{m-1}\right) \otimes_{\mathbb{R}} \mathbb{C}=W_{\lambda} \oplus W_{\bar{\lambda}} .
$$

Since $W_{\lambda}=\bar{W}_{\bar{\lambda}}$, the first Chern class vanishes:

$$
0=c_{1}\left(T\left(\mathbb{R} P^{m-1}\right) \otimes_{\mathbb{R}} \mathbb{C}\right) \in H^{2}\left(\mathbb{R} \mathbb{P}^{m-1} ; \mathbb{Z}_{2}\right)=\mathbb{Z}_{2}
$$

On the other hand, $\mathbb{R} \mathbb{P}^{m-1}$ is not orientable since $m-1$ is even. Thus $w_{1}\left(T\left(\mathbb{R P}^{m-1}\right)\right)$ generates $H^{1}\left(\mathbb{R P}^{m-1} ; \mathbb{Z}_{2}\right)=\mathbb{Z}_{2}$. Since the generator of the first cohomology 
group $H^{1}\left(\mathbb{R P}^{m-1} ; \mathbb{Z}_{2}\right)$ squares to the generator of the second cohomology group $H^{2}\left(\mathbb{R P}^{m-1} ; \mathbb{Z}_{2}\right)$, this implies

$$
0 \neq w_{1}^{2}\left(T\left(\mathbb{R} \mathbb{P}^{m-1}\right)\right) \in H^{2}\left(\mathbb{R} \mathbb{P}^{m-1} ; \mathbb{Z}_{2}\right)=\mathbb{Z}_{2} .
$$

This is a contradiction since

$$
w_{1}^{2}\left(T\left(\mathbb{R} \mathbb{P}^{m-1}\right)\right)=c_{1}\left(T\left(\mathbb{R} \mathbb{P}^{m-1}\right) \otimes_{\mathbb{R}} \mathbb{C}\right) .
$$

This contradiction completes the proof.

\section{The PRoof of TheOrem 1.11}

If $m=2$, then Theorem 1.11 follows from Theorem 1.9 (2) so we shall assume that $m \geq 3$. We have defined $\mathfrak{M}_{\varepsilon}:=\left(\mathbb{R}^{m}, A\right)$, where the non-zero components of $A$ are determined by:

$$
A_{i j j}{ }^{i}=1 \text { for } 1 \leq i \neq j \leq m \quad \text { and } \quad A_{122}{ }^{2}=A_{121}{ }^{1}=-\varepsilon .
$$

If $(\cdot, \cdot)$ is the usual Euclidean inner product on $\mathbb{R}^{m}$, then $\rho_{s}=(m-1)(\cdot, \cdot)$. We let $G:=S O(2) \times S O(m-2)$ act on $\mathbb{R}^{m}$. We lower indices and regard $A \in \otimes^{4} V^{*}$ :

$$
A=-\varepsilon\left(e^{1} \wedge e^{2}\right) \otimes\left(e^{1} \otimes e^{1}+e^{2} \otimes e^{2}\right)+\sum_{i<j}\left(e^{i} \wedge e^{j}\right) \otimes\left(e^{j} \wedge e^{i}\right) .
$$

Consequently $A$ is invariant under the action of $G$ so $\operatorname{Spec}\{\mathcal{J}(x)\}=\operatorname{Spec}\{\mathcal{J}(g x)\}$ for all $g \in G$. Let $x=a_{1} e_{1}+\ldots+a_{m} e_{m}$ belong to $S^{m-1}$. We may use the action of $S O(2)$ to ensure that $a_{2}=0$ and we may use the the action of $S O(m-2)$ to ensure that $a_{i}=0$ for $i>3$ in examining $\operatorname{Spec}\{\mathcal{J}(x)\}$. Thus we may assume that $x=\cos \theta e_{1}+\sin \theta e_{3}$ so

$$
\begin{aligned}
& \mathcal{J}(x) e_{i}=e_{i} \text { for } i \geq 4, \\
& \mathcal{J}(x)\left(\cos \theta e_{1}+\sin \theta e_{3}\right)=0, \\
& \mathcal{J}(x)\left(-\sin \theta e_{1}+\cos \theta e_{3}\right)=-\sin \theta e_{1}+\cos \theta e_{3} .
\end{aligned}
$$

Thus 0 is an eigenvalue of multiplicity at least 1 and +1 is a eigenvalue of multiplicity at least $m-2$. Since $\operatorname{Tr}\{\mathcal{J}(x)\}=\rho(x, x)=(m-1)$, we conclude that +1 is an eigenvalue of multiplicity $m-1$. Consequently, $\mathfrak{M}_{\varepsilon}$ is an affine projective Osserman curvature model for any $\varepsilon$.

Define a torsion free connection $\nabla$ on $\mathbb{R}^{m}$ by setting:

$$
\Gamma_{m m}^{m}=2, \Gamma_{i m}{ }^{i}=\Gamma_{m i}{ }^{i}=\Gamma_{i i}{ }^{m}=1 \text { for } i<m ; \Gamma_{11}{ }^{1}=-\Gamma_{22}{ }^{2}=\varepsilon\left(x_{1}+x_{2}\right) .
$$

We have $R_{i j k}{ }^{l}=\partial_{x_{i}} \Gamma_{j k}{ }^{l}-\partial_{j} \Gamma_{i k}{ }^{l}+\Gamma_{i n}{ }^{l} \Gamma_{j k}{ }^{n}-\Gamma_{j n}{ }^{l} \Gamma_{i k}{ }^{n}$. There are no terms in $\varepsilon^{2}$ and the only terms in $\varepsilon$ which are quadratic in the Christoffel symbols are

$$
\begin{aligned}
& 0=\Gamma_{m 1}{ }^{1} \Gamma_{11}{ }^{1}-\Gamma_{11}{ }^{1} \Gamma_{m 1}{ }^{1}=0, \\
& 0=\Gamma_{m 2}{ }^{2} \Gamma_{22}{ }^{2}-\Gamma_{22}{ }^{2} \Gamma_{m 2}{ }^{2}=0 .
\end{aligned}
$$

Consequently, the quadratic terms give rise to:

$$
\begin{aligned}
& R_{i m m}{ }^{i}=\Gamma_{i m}{ }^{i} \Gamma_{m m}{ }^{m}-\Gamma_{m i}{ }^{i} \Gamma_{i m}{ }^{i}=2-1 \text { for } i<m, \\
& R_{m i i}{ }^{m}=\Gamma_{m m}{ }^{m} \Gamma_{i i}{ }^{m}-\Gamma_{i i}{ }^{m} \Gamma_{m i}{ }^{i}=2-1 \text { for } i<m, \\
& R_{i j j}{ }^{i}=\Gamma_{i m}{ }^{i} \Gamma_{j j}{ }^{m}=1 \text { for } i \neq j<m .
\end{aligned}
$$

We complete the proof by examining the terms involving the derivatives of $\Gamma$ and verifying: 


$$
R_{122}^{2}=\partial_{x_{1}} \Gamma_{22}{ }^{2}=-\varepsilon \text { and } R_{211}{ }^{1}=\partial_{x_{2}} \Gamma_{11}{ }^{1}=\varepsilon .
$$

Remark 6.1. Suppose $\varepsilon \neq 0$. If $\theta=\frac{\pi}{2}$, then $x=e_{3}$ and $\mathcal{J}(x)$ is diagonal. If $\theta=\frac{\pi}{4}$, then $x=\frac{1}{\sqrt{2}}\left(e_{1}+e_{3}\right)$ and:

$$
\begin{aligned}
& \mathcal{J}(x)\left(e_{1}+e_{3}\right)=0, \quad \mathcal{J}(x)\left(e_{1}-e_{3}\right)=e_{1}-e_{3}, \quad \mathcal{J}(x) e_{2}=\frac{1}{2} \varepsilon e_{1}+e_{2}, \\
& \mathcal{J}(x)\left\{e_{2}+\frac{1}{4} \varepsilon\left(e_{1}+e_{3}\right)\right\}=\frac{1}{2} \varepsilon e_{1}+e_{2}=e_{2}+\frac{1}{4} \varepsilon\left(e_{1}+e_{3}\right)+\frac{1}{4} \varepsilon\left(e_{1}-e_{3}\right) .
\end{aligned}
$$

Thus the space $\operatorname{Span}\left\{u_{1}:=e_{2}+\frac{1}{4} \varepsilon\left(e_{1}+e_{3}\right), v_{2}=\frac{1}{4} \varepsilon\left(e_{1}-e_{3}\right)\right\}$ is invariant under the action of $\mathcal{J}(x)$ and we have $\mathcal{J}(x) v_{2}=v_{2}$ and $\mathcal{J}(x) v_{1}=v_{1}+v_{2}$. Consequently, we have non-trivial Jordan normal form in this instance.

Remark 6.2. Suppose that $\varepsilon=0$. Since the Christoffel symbols are constant, the group of translations acts on transitively on $\mathcal{M}$ by affine isomorphisms; thus $\mathcal{M}$ is affine homogeneous. However, if we set $\sigma(t)=(0, \ldots, 0, x(t))$, then the geodesic equation becomes $\ddot{x}+\dot{x} \dot{x}=0$ which blows up in finite time for suitable initial conditions. Thus $\left(\mathbb{R}^{m}, \nabla\right)$ is geodesically incomplete. Finally, we compute:

$$
\begin{aligned}
& \nabla R\left(\partial_{m}, \partial_{1}, \partial_{1} ; \partial_{1}\right) \\
= & \nabla_{\partial_{m}} R\left(\partial_{m}, \partial_{1}\right) \partial_{1}-R\left(\nabla_{\partial_{m}} \partial_{m}, \partial_{1}\right) \partial_{1}-R\left(\partial_{m}, \nabla_{\partial_{m}} \partial_{1}\right) \partial_{1}-R\left(\partial_{m}, \partial_{1}\right) \nabla_{\partial_{m}} \partial_{1} \\
= & (2-2-2) \partial_{m} \neq 0 .
\end{aligned}
$$

Consequently, $\nabla \mathcal{R} \neq 0$. Thus these manifolds are not locally symmetric. This shows that the affine manifolds $\mathcal{M}_{0}$ are not affinely equivalent to the standard affine structure on the sphere $S^{m}$.

If $\varepsilon \neq 0$, then there is a translation group of rank $m-1$ which acts on $(M, \nabla)$ preserving the structures. Furthermore, this manifold is affine curvature homogeneous. However, we have additional entries in $\nabla R$ :

$$
\nabla R\left(\partial_{2}, \partial_{1}, \partial_{1} ; \partial_{1}\right)=-2 \Gamma_{11}^{1} \partial_{2} \text {, and } \nabla R\left(\partial_{1}, \partial_{2}, \partial_{2} ; \partial_{2}\right)=-2 \Gamma_{22}^{2} \partial_{1} \text {. }
$$

Since $\Gamma_{11}{ }^{1}$ and $\Gamma_{22}{ }^{2}$ vanishe if and only if $x_{1}+x_{2}=0,(M, \nabla)$ is not 1-affine curvature homogeneous and has affine cohomogeneity 1.

\section{Two algebraic examples}

In Section 6, we considered a model based on the tensor of constant sectional curvature 1 . In this section, we examine examples which are related to the curvature operators of complex and projective space. These examples have non-symmetric Ricci tensors and non-trivial Jordan normal form. We do not know if any of the examples in this section can be realized geometrically.

7.1. A complex example. Let $m=2 \bar{m}$ be even, let $(\cdot, \cdot)$ be the usual positive definite inner product on $\mathbb{R}^{m}$ for $m$ even, and let $J$ be a Hermitian complex structure; this means that

$$
J^{*}(\cdot, \cdot)=(\cdot, \cdot) \text { and } J^{2}=-\mathrm{Id} .
$$

We can choose an orthonormal basis $\left\{e_{1}, \ldots, e_{m}\right\}$ for $\mathbb{R}^{m}$ so that if $1 \leq j \leq \bar{m}$, then:

$$
J e_{i}=\left\{\begin{array}{l}
e_{2 j} \text { if } i=2 j-1 \\
-e_{2 j-1} \text { if } i=2 j
\end{array}\right\} .
$$

Define algebraic affine curvature operators by setting:

$$
\begin{aligned}
& A_{0}(x, y) z:=(y, z) x-(x, z) y, \\
& A_{J}(x, y) z:=(J y, z) J x-(J x, z) J y-2(J x, y) J z, \\
& \mathcal{E}\left(e_{1}, e_{2}\right) e_{1}=-e_{1} \text { and } \mathcal{E}\left(e_{2}, e_{1}\right) e_{2}=e_{2} .
\end{aligned}
$$


The tensor $A_{0}+A_{J}$ is the curvature operator of the Fubini-Study metric on complex projective space $\mathbb{C P}^{\bar{m}}$. If $(x, x)=1$, then

$$
\mathcal{J}_{\lambda_{0} A_{0}+\lambda_{1} A_{J}}(x) \cdot y=\left\{\begin{array}{rll}
0 & \text { if } & y=x \\
\left(\lambda_{0}+3 \lambda_{1}\right) y & \text { if } & y=J x \\
\lambda_{0} y & \text { if } & y \perp\{x, x\}
\end{array}\right\} .
$$

Thus $\lambda_{0} A_{0}+\lambda_{1} A_{J}$ is an affine projective Osserman curvature model.

Lemma 7.1. Let $\mathfrak{M}_{\varepsilon}:=\left(\mathbb{R}^{m}, \lambda_{0} A_{0}+\lambda_{1} A_{J}+\varepsilon \mathcal{E}\right)$. The eigenvalues of $\mathcal{J}(x)$ for $(x, x)=1$ are $\left\{0, \lambda_{0}+3 \lambda_{1}, \lambda_{0}, \ldots, \lambda_{0}\right\}$ where each eigenvalue is repeated according to multiplicity. Thus $\mathfrak{M}_{\varepsilon}$ is an affine projective Osserman curvature model.

Proof. The tensor $A_{0}$ is invariant under the action of the full orthogonal group $O(m)$, the tensor $A_{J}$ is invariant under the action of the unitary group $U(\bar{m})$, and the tensor $\mathcal{E}$ is invariant under the action of the group

$$
G:=U(1) \times U(\bar{m}-1) \subset U(\bar{m}) \subset O(m) .
$$

We suppose $x_{1} \in \mathbb{R}^{m}$ satisfies $\left(x_{1}, x_{1}\right)=1$. We use the action of $G$ to assume $x_{1}=\cos \theta e_{1}+\sin \theta e_{3}$ when studying $\mathcal{J}(x)$. Then $\mathcal{J}(x)=\operatorname{Id}$ on $\operatorname{Span}\left\{e_{i}\right\}_{i \geq 5}$ so this space plays no role and we may assume $m=4$. Let

$$
\begin{array}{ll}
x_{1}:=\cos \theta e_{1}+\sin \theta e_{3}, & x_{2}:=-\sin \theta e_{1}+\cos \theta e_{3}, \\
x_{3}:=J x_{1}=\cos \theta e_{2}+\sin \theta e_{4}, & x_{4}:=J x_{2}=-\sin \theta e_{2}+\cos \theta e_{4} .
\end{array}
$$

Let $\star$ be a coefficient which we do not need to specify. We then have

$$
\begin{aligned}
& \mathcal{J}\left(x_{1}\right) x_{1}=0, \\
& \mathcal{J}\left(x_{1}\right) x_{2}=\lambda_{0} x_{2}, \\
& \mathcal{J}\left(x_{1}\right) x_{3}=\left(\lambda_{0}+3 \lambda_{1}\right) x_{3}+\star e_{1}=\star x_{1}+\star x_{2}+\left(\lambda_{0}+3 \lambda_{1}\right) x_{3}, \\
& \mathcal{J}\left(x_{1}\right) x_{4}=\lambda_{0} x_{4}+\star e_{1}=\star x_{1}+\star x_{2}+\lambda_{0} x_{4} .
\end{aligned}
$$

The matrix of $\mathcal{J}\left(x_{1}\right)$ on this 4 -dimensional subspace is therefore given by

$$
\mathcal{J}\left(x_{1}\right)=\left(\begin{array}{llll}
0 & 0 & \star & \star \\
0 & \lambda_{0} & \star & \star \\
0 & 0 & \lambda_{0}+3 \lambda_{1} & 0 \\
0 & 0 & 0 & \lambda_{0}
\end{array}\right) .
$$

The Lemma now follows.

Remark 7.2. If we take $\theta=\frac{\pi}{2}$, then $x_{1}=e_{3}$ and $\mathcal{J}\left(x_{1}\right)$ is diagonal. If we take $\theta=\frac{\pi}{4}$, then $x_{1}=\left(e_{1}+e_{3}\right) / \sqrt{2}$ and the same argument given in Remark 6.1 shows $\mathcal{J}\left(x_{1}\right)$ has non-trivial Jordan normal form:

$$
\begin{gathered}
\mathcal{J}(x)\left(e_{1}+e_{3}\right)=0, \quad \mathcal{J}(x)\left(e_{1}-e_{3}\right)=e_{1}-e_{3}, \\
\mathcal{J}(x)\left(e_{2}-e_{4}\right)=\frac{1}{2} \varepsilon e_{1}+\left(e_{2}-e_{4}\right), \\
\mathcal{J}(x)\left\{e_{2}-e_{4}+\frac{1}{4} \varepsilon\left(e_{1}+e_{3}\right)\right\}=\frac{1}{2} \varepsilon e_{1}+e_{2}-e_{4} \\
=e_{2}-e_{4}+\frac{1}{4} \varepsilon\left(e_{1}+e_{3}\right)+\frac{1}{4} \varepsilon\left(e_{1}-e_{3}\right) .
\end{gathered}
$$

and, again, the Jordan normal form is non-trivial if $\varepsilon \neq 0$.

7.2. A quaternion example. Let $m=4 k$ and let $\left\{J_{1}, J_{2}, J_{3}\right\}$ give $\mathbb{R}^{4 k}$ an orthogonal quaternion structure, i.e.

$$
\left(J_{i} x, J_{i} x\right)=(x, x), \quad J_{i} J_{j}+J_{j} J_{i}=-2 \delta_{i j} \mathrm{Id}, \text { and } J_{1} J_{2}=J_{3} .
$$

Let

$$
\mathcal{E}:=-\left\{\left(e^{1} \wedge e^{2}\right) \otimes\left(e^{1} \otimes e^{1}+e^{2} \otimes e^{2}\right)+\left(e^{3} \wedge e^{4}\right)\left(e^{3} \otimes e^{3}+e^{4} \otimes e^{4}\right)\right\}
$$


Lemma 7.3. Let $\mathfrak{M}_{\varepsilon}:\left(\mathbb{R}^{m}, \lambda_{0} A_{0}+\lambda_{1} A_{J_{1}}+\lambda_{2} A_{J_{2}}+\lambda_{3} A_{J_{3}}+\varepsilon \mathcal{E}\right)$. The eigenvalues of $\mathcal{J}(x)$ for $(x, x)=1$ are $\left\{0, \lambda_{0}+3 \lambda_{1}, \lambda_{0}+3 \lambda_{2}, \lambda_{0}+3 \lambda_{3}, \lambda_{0}, \ldots, \lambda_{0}\right\}$ where each eigenvalue is repeated according to multiplicity. Thus $\mathfrak{M}_{\varepsilon}$ is an affine projective Osserman curvature model.

Proof. Let $\mathbb{H}:=\operatorname{Span}_{\mathbb{R}}\{1, i, j, k\}$ denote the quaternions. We take an orthonormal basis $\left\{e_{1}^{\nu}, e_{i}^{\nu}, e_{j}^{\nu}, e_{k}^{\nu}\right\}$ for $\mathbb{R}^{4 k}$ where $1 \leq \nu \leq k$ so that $e_{i}^{\nu}=J_{1} e_{1}^{\nu}, e_{j}^{\nu}=J_{2} e_{1}^{\nu}$, and $e_{k}^{\nu}=J_{3} e_{1}^{\nu}$. This permits us to identify $\mathbb{R}^{m}=\mathbb{H}^{k}$ with the quaternions where $\left\{J_{1}=i, J_{2}=j, J_{3}=k\right\}$ are the quaternions acting from the left. Let $\operatorname{Sp}(k)$ be the group of isometries of $\mathbb{R}^{m}$ which commute $\left\{J_{1}, J_{2}, J_{3}\right\}$; this is the set of $k \times k$ orthogonal quaternion matrices acting from the right. The affine algebraic curvature tensor in question is invariant under the action of $\operatorname{Sp}(1) \times \operatorname{Sp}(k-1)$. Consequently, in considering $\operatorname{Spec}\{\mathcal{J}(x)\}$, it suffices to consider the special case $x=\cos \theta e_{1}^{1}+\sin \theta e_{1}^{2}$. The remaining variables $e_{*}^{\nu}$ for $\nu \geq 3$ play no role and may be ignored. We compute:

$$
\begin{aligned}
& \mathcal{J}(x)\left(\cos \theta e_{1}^{1}+\sin \theta e_{1}^{2}\right)=0, \\
& \mathcal{J}(x)\left(-\sin \theta e_{1}^{1}+\cos \theta e_{1}^{2}\right)=\lambda_{0}\left(-\sin \theta e_{1}^{1}+\cos \theta e_{1}^{2}\right), \\
& \mathcal{J}(x)\left(\cos \theta e_{i}^{1}+\sin \theta e_{i}^{2}\right)=\left(\lambda_{0}+3 \lambda_{1}\right)\left(\cos \theta e_{i}^{1}+\sin \theta e_{i}^{2}\right)+\star e_{1}^{i}, \\
& \left.\mathcal{J}(x)\left(-\sin \theta e_{i}^{1}+\cos \theta e_{i}^{2}\right)=\lambda_{0}\left(-\sin \theta e_{i}^{1}+\cos \theta e_{i}^{2}\right)+\star e_{1}^{i}\right), \\
& \mathcal{J}(x)\left(\cos \theta e_{j}^{1}+\sin \theta e_{j}^{2}\right)=\left(\lambda_{0}+3 \lambda_{2}\right)\left(\cos \theta e_{j}^{1}+\sin \theta e_{j}^{2}\right), \\
& \mathcal{J}(x)\left(-\sin \theta e_{j}^{1}+\cos \theta e_{j}^{1}\right)=\lambda_{0}\left(-\sin \theta e_{j}^{2}+\cos \theta e_{j}^{2}\right), \\
& \mathcal{J}(x)\left(\cos \theta e_{k}^{1}+\sin \theta e_{k}^{2}\right)=\left(\lambda_{0}+3 \lambda_{1}\right)\left(\cos \theta e_{k}^{1}+\sin \theta e_{k}^{2}\right), \\
& \mathcal{J}(x)\left(-\sin \theta e_{k}^{1}+\cos \theta e_{k}^{1}\right)=\lambda_{0}\left(-\sin \theta e_{k}^{2}+\cos \theta e_{k}^{2}\right) .
\end{aligned}
$$

The last 4 vectors play no role and the matrix of $\mathcal{J}(x)$ with respect to the first 4 vectors takes the form:

$$
\mathcal{J}(x)=\left(\begin{array}{rrrr}
0 & 0 & \star & \star \\
0 & \lambda_{0} & \star & \star \\
0 & 0 & \lambda_{0}+3 \lambda_{1} & 0 \\
0 & 0 & 0 & \lambda_{0}
\end{array}\right) .
$$

The desired result now follows.

7.3. Acknowledgments. The research of the authors was partially supported by Project MTM2009-07756 (Spain) and by Project 174012 (Srbija). The paper is dedicated to our friend and colleague Franki Dillen; may he rest in peace.

\section{REFERENCES}

[1] M. Brozos-Vázquez and E. Merino, "Equivalence between the Osserman condition and the Rakić duality principle in dimension 4", J. Geom. Phys. 62 (2012), no. 12, 2346-2352.

[2] Y. Euh, P. Gilkey, J.H. Park, and K. Sekigawa, "Transplanting geometric structures", Differential Geom. Appl. 31 (2013) 374-387.

[3] M. Brozos-Vázquez, M. E. Vázquez-Abal, and R. Vázquez-Lorenzo, "Conformally Osserman multiply warped product structures in the Riemannian setting", Differential geometry, 185194, World Sci. Publ., Hackensack, NJ, 2009.

[4] E. Calvino-Louzao, E. García-Río, P. Gilkey, and R. Vázquez-Lorenzo, "The geometry of modified Riemannian extensions", Proc. R. Soc. A. 465 (2009), 2023-2040.

[5] E. Calvino-Louzao, E. García-Río, P. Gilkey, and R. Vázquez-Lorenzo, "Higher dimensional Osserman metrics with non-nilpotent Jacobi operators", Geom. Dedicata 156 (2012) 151-163.

[6] E. Calviño-Louzao, E. García-Río, and R. Vázquez-Lorenzo, "Four-dimensional Osserman Ivanov Petrova metrics of neutral signature", Class. Quantum Grav. 24 (2007), 2343-2355.

[7] M. Chaichi, E. Garca-Ro, and Y. Matsushita, "Curvature properties of four-dimensional Walker metrics", Classical Quantum Gravity 22 (2005), no. 3, 559-577.

[8] A. Chudecki and M. Przanowski, "From hyperheavenly spaces to Walker and Osserman spaces I", Classical Quantum Gravity 25 (2008), no. 14, 145010, 18 pp. 
[9] A. Chudecki and M. Przanowski, "From hyperheavenly spaces to Walker and Osserman spaces II", Classical Quantum Gravity 25 (2008), no. 23, 235019, 22 pp.

[10] Q. S. Chi, "A curvature characterization of certain locally rank-one symmetric spaces", J. Diff. Geom. 28 (1988), 187-202.

[11] A. Derdzinski, "Non-Walker self-dual neutral Einstein four-manifolds of Petrov type III", J. Geom. Anal. 19 (2009), 301-357.

[12] J.C. Díaz-Ramos, E. Garca-Río, and R. Vázquez-Lorenzo, "Four-dimensional Osserman metrics with nondiagonalizable Jacobi operators", J. Geom. Anal. 16(2006), 39-52.

[13] E. García-Río, P. Gilkey, S. Nikčević, and R. Vázquez-Lorenzo, "Applications of Affine and Weyl Geometry", forthcoming from Morgan \& Claypool.

[14] J. C. Díaz-Ramos, E. García-Río, and R. Vázquez-Lorenzo, "New examples of Osserman metrics with nondiagonalizable Jacobi operators", Differential Geom. Appl. 24 (2006), 433442.

[15] E. García-Río, D. N. Kupeli, and M. E. Vázquez-Abal, "On a problem of Osserman in Lorentzian geometry", Differential Geom. Appl. 7 (1997), 85-100.

[16] E. García-Río, D. N. Kupeli, M. E. Vázquez-Abal, and R. Vázquez-Lorenzo, "Affine Osserman connections and their Riemann extensions", Differential Geom. Appl. 11 (1999), 145-153.

[17] P. Gilkey and R. Ivanova, "The Jordan normal form of Osserman algebraic curvature tensors", Results Math. 40 (2001), 192-204.

[18] P. Gilkey, A. Swann, and L. Vanhecke, "Isoparametric geodesic spheres and a conjecture of Osserman concerning the Jacobi operator", Quart. J. Math. Oxford 46 (1995), 299-320.

[19] Y. Nikolayevsky, "Osserman manifolds of dimension 8", Manuscripta Math. 115 (2004), 3153.

[20] Y. Nikolayevsky, "Osserman conjecture in dimension $\neq$ 8, 16", Math. Ann. 331 (2005), 505522 .

[21] Y. Nikolayevsky, "On Osserman manifolds of dimension 16", Contemporary geometry and related topics Univ. Belgrade Fac. Math., Belgrade, (2006), 379-398.

[22] Y. Nikolayevsky, "Conformally Osserman manifolds of dimension 16 and a Weyl-Schouten theorem for rank-one symmetric spaces", Ann. Mat. Pura Appl. 19 (2012), 677-709

[23] R. Osserman, "Curvature in the eighties", Amer. Math. Monthly 97 (1990), 731-756.

PG: Mathematics Department, University of Oregon, Eugene OR 97403 USA E-mail address: gilkey@uoregon.edu

SN: Mathematical Institute, Sanu, Knez Mihailova 36, P.P. 367, 11001 Belgrade, SERBIA

E-mail address: stanan@mi.sanu.ac.rs 